\title{
Bringing phylogeny and clinical microbiology together
}

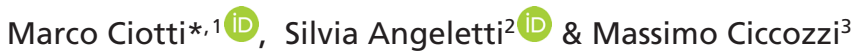 \\ ${ }^{1}$ Laboratory of Microbiology and Virology, Polyclinic Tor Vergata Foundation, Viale Oxford 81, 00133 Rome, Italy \\ ${ }^{2}$ Unit of Clinical Laboratory Science, University Campus Bio-Medico of Rome, Rome, Italy \\ ${ }^{3}$ Unit of Medical Statistics and Molecular Epidemiology, University Campus Bio-Medico of Rome, Rome, Italy \\ *Author for correspondence: Tel.: +39 062090 2087; marco.ciotti@ptvonline.it
}

'In recent years, phylogenetic and phylodynamic analyses combined to WGS by next-generation sequencing have been successfully applied to the epidemiological surveillance of multidrug-resistant nosocomial pathogens."

First draft submitted: 25 September 2019; Accepted for publication: 19 December 2019; Published online: 11 February 2020

Keywords: antibiotic resistance $\bullet$ bacterial species $\bullet$ clustering $\bullet$ community outbreak $\bullet$ Enterobacteriaceae $\bullet$ intraspecies variation • MALDI-TOF MS • NGS • nosocomial outbreak • phylogenesis

\section{Why are new methods to detect resistance needed?}

Antibiotic resistance occurs naturally, but the misuse of antibiotics at a global level has accelerated and facilitated the spreading of the resistant microorganisms through their selection, making more difficult to treat the infections. This has translated into an increased mortality and morbidity; longer hospital stays and higher costs for the health services. Therefore, it is of paramount importance to prevent infections and control the spread of resistant strains within the community and hospital wards where the majority of resistant infections occur [1,2].

Phenotypic (i.e, minimum inhibitory concentration and microdilution broth) and molecular methods (i.e., pulsed field gel electrophoresis, multilocus sequence typing and whole genome sequencing [WGS] are currently available to detect the presence of resistant microorganisms).

Classical phenotypic methods require at least $36-48 \mathrm{~h}$ for delivering a clinical report, thus delaying the administration of an optimal and effective antibiotic therapy with consequent impact on patient survival in the presence of severe infections.

Molecular methods not requiring culture growth of microorganisms are faster and represent an advantage over the traditional methods. Also, they require skilled personnel and economic resources not always available in the laboratories of clinical microbiology, thus limiting their diffusion.

\section{MALDI-TOF-MS for microbial identification}

Recently, MALDI-TOF MS is gaining an increasingly important role in the determination of antibiotic resistance as well as in the identification of pathogens. MALDI-TOF MS, combining MALDI source and the TOF, has largely contributed to microbial identification improvement in routine microbiology, becoming a reference method for the identification of the most important microbial pathogens [3]. Furthermore, MALDI-TOF MS measuring mass differences can quickly reveal the presence of bacterial strains producing enzymes that are able to degrade the given antibiotic. For instance, hydrolysis of $\beta$-lactam ring causes a mass shift of $+18 \mathrm{Da}$ that is easily detected by this technology, demonstrating the production of $\beta$-lactamases by the resistant strains $[4,5]$. Similarly, carbapenemaseproducing and noncarbapenemase-producing strains can be quickly detected by MALDI-TOF MS technology and discriminated based on the different proteomic profiles [6].

\section{Phylogenetic \& phylogeographic analysis}

In recent years, phylogenetic and phylodynamic analyses combined to WGS by next-generation sequencing have been successfully applied to the epidemiological surveillance of multidrug-resistant nosocomial pathogens. This

Future $\because$ Medicine 
has been nicely shown in the work by Cella et al. [7], where it was demonstrated that multidrug-resistant Klebsiella pneumoniae strains circulating among the hospital wards between 2012 and 2013 were most likely introduced into the hospital in 2007, as suggested by the time-scaled phylogeny. Phylogeny also demonstrated that two distinct epidemic introductions occurred in 2008 and 2009. Furthermore, the Bayesian phylogenetic and phylogeographic analyses were used to study the spread of $K$. pneumoniae strains in different hospital wards and at different times. Based on the analyses it was possible to assume which hospital ward the pathogen started spreading from. Thus, allowing the hospital staff to put in place measures aimed at preventing and/or reducing the risk of diffusion of the pathogen in the wards.

Another significant example of the importance of the application of the phylogenetic methods to the study and control of the spread of multidrug-resistant K. pneumoniae strains, is given by the study of Ciccozzi et al. [8], performed on the strains of $K$. pneumoniae isolated from duodenoscope biofilm. In this case, phylogenetic analysis of the WGS of strains isolated from duodenoscope and the inpatients was conducted. Multidrug-resistant strains showed intermixing between duodenoscope and inpatients as shown by minimum spanning tree and time-scale Maximum Clade Credibility Tree. The minimum spanning tree revealed the presence of three groups of K. pneumoniae strains. Group 1 comprised K. pneumoniae strains identified in inpatients before the adoption of microbiological surveillance; group 2 contained mixed K. pneumoniae strains isolated both from inpatients and duodenoscopes, whereas group 3 included K. pneumoniae strains isolated from duodenoscopes only. The maximum credibility tree showed a statistically supported cluster containing two $K$. pnuomoniae strains coming from duodenoscopes and one strain isolated from inpatients. Taken together, these results suggest the importance of performing a microbiological surveillance on the duodenoscope instruments, as well as inpatients by rectal swabs culture. Furthermore, a rapid molecular testing for antimicrobial resistance is suggested before any invasive endoscopic procedures.

\section{Is combining the two techniques the answer?}

Clustering analysis performed by MALDI-TOF MS has been used to demonstrate the circulation of Enterobacter species between hospital wards including multidrug-resistant strains [9].

Phylogenetic analysis is also used to determine the level of relatedness between microbial isolates based on gene sequence. The two different approaches can be used in a complementary way to identify the origin of an outbreak within a hospital setting. This is exemplified by the work of Batah et al. [10] who reported an outbreak of Serratia marcescens strains in eastern Algeria between December 2011 and July 2013. The results obtained by MALDI-TOF MS clustering were compared to those obtained by phylogenetic analysis. Both methods confirmed the presence of a cluster of extended-spectrum $\beta$-lactamase isolates that originated from the same ward. Thus, suggesting that they are both useful for outbreak identification.

Similarly, MALDI-TOF clustering and phylogenetic analysis were successfully applied in the characterization of an outbreak of carbapenem-resistant $K$. pneumoniae strains. Two clusters were identified by MALDI-TOF clustering characterized by a defined temporal split: cluster 1 contained strains isolated in 2012, while strains of cluster 2 were isolated from the end of 2012 to the beginning of 2013. This clustering analysis was confirmed by phylogenetic analysis. In particular, phylogenetic analysis applied to bla-KPC gene confirmed the genetic diversity of strains included in the two clusters. This study confirmed that MALDI-TOF is a reliable method for epidemiological investigation in a nosocomial setting and can be used in combination with phylogenetic analysis for clonal strains identification [11]. With a similar approach, combining MALDI-TOF MS and Bayesian phylogenetic analysis were characterized clinical isolates of Candida species [12].

This brief overview of the recent scientific literature shows the usefulness of the MALDI-TOF MS in the identification, antibiotic susceptibility and clustering analysis of pathogen microorganisms responsible for outbreaks, especially in nosocomial settings. The method is rapid, effective and cost saving, and it has been successfully introduced in the routine work flow of clinical microbiology laboratories. MALDI-TOF MS used in combination with phylogenetic analysis will improve the control and surveillance of multidrug-resistant pathogens. The information derived from the phylogenetic analysis can trace the spread of pathogen microorganisms and may help to implement prevention measures to at reduce the risk of outbreaks within nosocomial settings. This is in addition to contributing to the genetic characterization of antibiotic-resistant pathogens. 


\section{Future perspective}

It can be envisaged that in the next coming years, phylogenetics and bacterial WGS will have an increasingly important role in the study of antibiotic resistance, development of multidrug-resistant microorganisms, their evolution and spreading.

This combined approach will allow researchers to obtain useful and detailed information about community and nosocomial outbreaks, the way the infection is transmitted and the genes responsible for the antibiotic resistance. The high-throughput sequencing will allow a faster identification of bacterial species and higher resolution of intraspecies variations. Furthermore, the generated sequencing data will improve patient management and guide the clinicians in the choice of the most effective therapy; thus, reducing the chance of selecting resistant microorganisms. In conclusion, these investigational approaches will support the health authorities in their battle against antibiotic resistance.

\section{Financial \& competing interests disclosure}

The authors have no relevant affiliations or financial involvement with any organization or entity with a financial interest in or financial conflict with the subject matter or materials discussed in the manuscript. This includes employment, consultancies, honoraria, stock ownership or options, expert testimony, grants or patents received or pending, or royalties.

No writing assistance was utilized in the production of this manuscript.

\section{References}

1. Roca MA, Baquero F, Carlet J et al. The global threat of antimicrobial resistance: science for intervention. New Microbes New Infect. 6, 22-29 (2015).

2. Azarian T, Maraqa NF, Cook RL et al. Genomic epidemiology of methicillin-resistant Staphylococcus aureus in a neonatal intensive care unit. PLoS ONE 11(10), e0164397 (2016).

3. Angeletti S. Matrix assisted laser desorption time of flight mass spectrometry (MALDI-TOF MS) in clinical microbiology. J. Microbiol. Methods 138, 20-29 (2017).

4. Sparbier K, Schubert S, Weller U, Boogen C, Kostrzewa M. Matrix-assisted laser desorption ionization-time of flight mass spectrometry-based functional assay for rapid detection of resistance against beta lactam antibiotics. J. Clin. Microbiol. 50(3), 927-937 (2012).

5. Jung JS, Popp C, Sparbier K, Lange C, Kostrzewa M, Schubert S. Evaluation of matrix-assisted laser desorption ionization-time of flight mass spectrometry for rapid detection of b-lactam resistance in Enterobacteriaceae derived from blood cultures. J. Clin. Microbiol. 52(3), 924-930 (2014).

6. Sakarikou C, Ciotti M, Dolfa C, Angeletti S, Favalli C. Rapid detection of carbapenemaseproducing Klebsiella pneumoniae strains derived from blood cultures by matrix-assisted laser desorption ionization-time of flight mass spectrometry (MALDI-TOF MS). BMC Microbiology 17(1), 54 (2017).

7. Cella E, Ciccozzi M, Lo Presti A et al. Multi-drug resistant Klebsiella pneumoniae strains circulating in hospital setting: wholegenome sequencing and Bayesian phylogenetic analysis for outbreak investigations. Sci. Rep. 7(1), 3534 (2017).

8. Ciccozzi M, Cella E, Lai A et al. Phylogenetic analysis of multi-drug resistant Klebsiella pneumoniae strains from duodenoscope biofilm: microbiological surveillance and reprocessing improvements for infection prevention. Front. Public Health 7, 219 (2019).

9. De Florio L, Riva E, Giona A et al. MALDI-TOF MS identification and clustering applied to Enterobacter species in nosocomial setting. Front. Microbiol. 9, 1885 (2018).

10. Batah R, Loucif L, Olaitan AO, Boutefnouchet N, Allag H, Rolain JM. Outbreak of Serratia marcescens coproducing ArmA and CTX-M-15 mediated high levels of resistance to aminoglycoside and extended-spectrum beta-lactamases, Algeria. Microb. Drug Resist. 21(4), 470-476 (2015).

11. Angeletti S, Dicuonzo G, Lo Presti A et al. MALDI-TOF mass spectrometry and blakpc gene phylogenetic analysis of an outbreak of carbapenem-resistant K. pneumoniae strains. New Microbiol. 38(4), 541-550 (2015).

12. Angeletti S, Lo Presti A, Cella E et al. Matrix-assisted laser desorption/ionization time of flight mass spectrometry (MALDI-TOF MS) and Bayesian phylogenetic analysis to characterize Candida clinical isolates. J. Microbiol. Methods 119, 214-222 (2015). 
